\title{
Interactive Emulator System to Aid Clinical Practice Outcomes for Nurses
}

\author{
http://dx.doi.org/10.3991/ijoe.v9iS5.2771 \\ Les Bowtell, Alexander A. Kist, Daniel Osbourne, Victoria R. Parker \\ University of Southern Queensland, Toowoomba, Australia
}

\begin{abstract}
Clinical practice is a key element of nursing studies programmes. Amongst its aims are familiarisation with medical equipment, appropriate use of clinical reasoning in a ward setting and calculation and delivery of patient medication fluids requirements. The setting for this is typically a simulated ward environment and is structured around group training. While this is cost effective, it does not cater well for minority groups and those wishing to train at their own pace. A number of group dynamic related social issues can also lead to students dominating others within the session. This can result in less than satisfactory learning outcomes for the remainder of the group. Other factors that come into play are the wide age range, social and ethnic backgrounds of the nursing student cohort.
\end{abstract}

Recent Remote Access Nursing Laboratory work has shown a marked improvement in residential school assessment results in areas such as readiness to practice and clinical reasoning levels demonstrated for those students in the trial nursing RAL project. With the aim of further enhancing the effectiveness and at the same time reducing implementation costs an interactive IV pump emulator was developed. Enhancements included guided introductions, provision of additional scaffolding and inbuilt annotated assessments. This paper discusses the techniques used and general implementation of this system and reports on positive results of the conducted trials.

Index Terms - Clinical Practice, Remote Access Laboratories, Nursing, Emulation, automation

\section{INTRODUCTION}

Opportunities for nursing students to practice clinical reasoning skills and to familiarise themselves with medical equipment are essential in nursing education. Traditionally training opportunities are largely based on faceto-face practical training sessions in simulated ward environments. Time on task in these situations is largely limited to classes and students have shown great difficulties in mastering tasks and equipment in the limited time available in practice sessions.

These issues are compounded for distance education students. At this university, for example, practical classes for nursing and midwifery students are limited to annual or bi-annual residential school classes on campus. Besides the limited access issues, there are potentially other problems with group training sessions. These include social challenges, difference in learning styles and pace, some students dominating others, limited opportunities for all group members to interact with the equipment, and social as well as ethnic backgrounds.
Remote Laboratories (RL) or Remote Access Laboratories (RAL) have been discussed in the context of science and engineering education for two decades $[1,2]$ as a way to allow offsite access to equipment and enable students to practice practical skills. The Faculty of Engineering and Surveying has developed a RAL system that allows for (off-campus, external) student to access hardware and software experiments on campus. A recent study into the use of RAL activities in non-engineering discipline has supported the development of a number of learning activities. In the discipline of nursing and midwifery an IV Pump RAL learning activity was developed [3].

In the pilot activity students control the system via screen interface mimics of a commonly used IV infusion pump. Inputs from the interface are used to control a peristaltic pump to deliver prescribed rates and volumes. Testing with students raised the question as to whether the inclusion of the physical pump had added any value to the learning activity. To investigate this aspect further this project has taken the approach of a completely emulated interface. The interface still looks the same but it no longer controls any hardware components. Student access is provided via the RAL environment with directly integrates with the learning management system, Moodle. The main aim of the learning activity is to address issues that were identified by students undertaking the previous study. Problem areas include: screen navigation, data entry, correct fluid calculations, entry of medication volumes and rates using a standard fluid chart.

A learning environment was develop to address these issues and a number of features were added to the learning activity include a guided learning mode, integrated resources, and a self-assessment mode. This paper addresses the research question of how well an emulated RAL IV pump activity can support skills acquisition for nursing students and whether an emulated RAL activity can better address intended learning outcomes than group hands on training. This paper extends initial results that were reported in [4]. Key contributions of this work include a detailed description of the learning activity design and initial evaluation results highlighting students learning outcomes of this novel activity.

The reminder of the paper is organised as follows: Section II discusses relates work and introduces the framework that underpins this project. Section III describes the learning activity and Section IV discusses the implementation in detail. Section V introduces the evaluation methodology and Section VI discusses results and findings. The paper concludes with Section VII. 


\section{THEORETICAL FRAMEWORK}

Initiatives in remote laboratories where first reported in the nineties in the context of remotely controlled hardware [1], [2]. Technologies to provide offsite access to equipment have been discussed for science and engineering since these early proposals, however, these technologies are not routinely used either in engineering or in other disciplines. In an attempt to make the affordances these technologies provide accessible to other disciplines, Kist et al. [5] has extended the traditional concept of RAL as purely controlling hardware to remote conceptual experimentation in any form. The laboratory experiments are seen as a way to create "episodes:" "recollections of events in which the (learner) took part or at least observed," with the result that the experience is "linked to propositions (about facts, concepts, ideas) so that those propositions in turn are remembered and understood" $[6$, pp.765-766]. Remote laboratories are events that help students to develop a better understanding of concepts by creating a (practical) learning experience via remote interfaces. This aspect is particularly important in the context of this paper as it evaluates an interactive emulator of an IV infusion pump and not a physical device which has been done in a previous study [3]. Barak's [7] proposes instructional principles about the effective design and use of ICT supported learning. These principles apply to this case as well. The principles are based on behavioral, cognitive and social learning theory and include: "learning is contextual, learning is an active process, learning is a social process, reflective practice plays a central role in learning" [7, pp.122-123] .

An "environment, in which the pupil can construct knowledge and can reflect upon his interactions and thinking" is important [8] and enforces that "learning implies the initiation of a thinking process" [p. 228]. Slangen \& Sloep [8] citing Jonassen [9] highlight three thinking tasks, i.e. basic, critical and creative thinking that are part of a complex thinking process. "Mind tools are computer applications that, when used by learners to represent what they know, necessarily engage them in critical thinking about the content they are studying ..." [10] and "they require students to think about what they know in different, meaningful ways" [pp.24]. The IV pump emulator discussed in this paper can be seen as an mind tool form an educational perspective.

Remote simulations in nursing education have been discussed as teaching strategies to improve the clinical reasoning skills of students [11-13]. Besides the obvious educational advantages, the most important reason for implementing simulated learning programs in nurse education has been identified by Messmer [14] as reducing patient death rates. The author suggests that technologies can prepare nursing students better to perform competently in stressful situations [14].

The key advantages of simulated clinical situations include the limited safety concerns where instructors are able to completely control the situation; examples include fully synthesized, robotic simulations $[15,16]$. Such virtual simulations allow students to gain competence by practicing skills over and over without patient risk [15]. Simulations in this context are different from simulations in the context of engineering as situations are simulated not systems. Remote access technology in nursing and healthcare is largely focussing on remote robotic presence

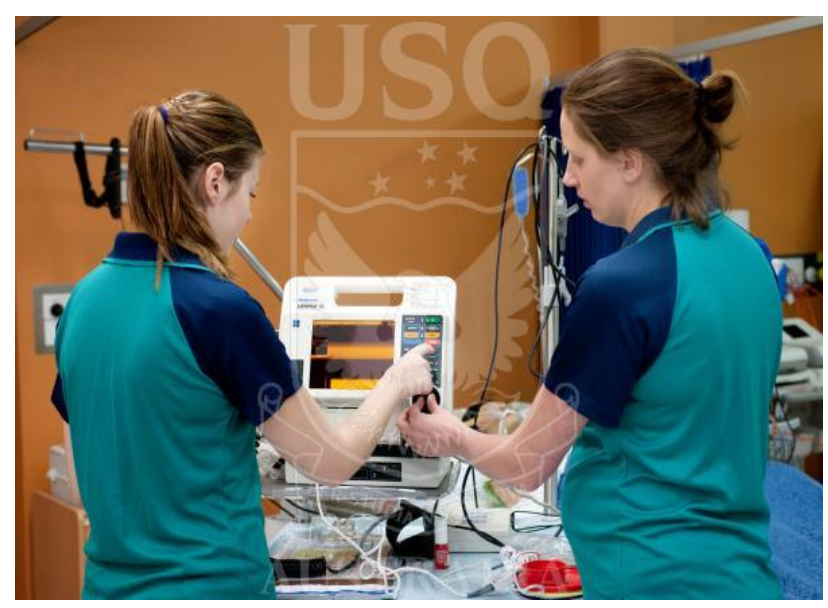

Figure 1. $1^{\text {st }}$ year nursing students in a simulated ward

technologies $[17,18]$. Using RAL technology in conjunction with an IVPE pump emulator allows for learning activities that achieve similar learning outcomes as have been highlighted in context of simulations in nursing education.

\section{IVPE PUMP DRIVER LEARNING ACTIVITY}

Setting up and monitoring an IV pump is an integral part of nursing practice. Figure 1 shows a typical simulated ward scenario used for first year nursing students at a residential school class. Typical components of a patient's medication chart such as an intravenous fluids form which shows the prescribed rates for the patient are used in these clinical scenarios. The role of the nurse is to correctly interpret this fluids order and program into the IV pump the necessary volume and flow-rate settings. Key skills being assessed are clinical reasoning by application of the fluids order to the prospective patient. The testing of these clinical reasoning skills includes taking appropriate remedial action in a timely fashion after correctly evaluating a patient's respiratory and cardiovascular clinical observations. At the same time the student must pay strict attention to observance of a patient's rights.

Throughout the various stages of this project [3], target learning areas were identified and solutions sought and trialed.

Key problem areas identified included:

- Pump Hardware interface issues

- Correctly converting standard patient medication fluids order details into their corresponding delivery volume and flow-rate figures for the IV infusion pump.

- Low levels of student confidence and interaction during an infusion to include critical responses to patient observations.

The first stage hardware based IV pump simulator was developed to specifically address these target areas and following on from this the IVPE was developed to include further enhancements and scaffolding not included in the initial system.

\section{LEARNING ACTIVITY ENHANCEMENTS}

While the initial hardware emulator system was successful in faithfully reproducing the IV Pump characteris- 
tics there were a number of areas identified where the students were still having difficulty and the IVPE's design was enhanced to specifically target those areas.

These additional areas included:

- A ground up demonstration for those students with little or no prior knowledge of the IV pump.

- Scaffolding material to aid all aspects of the IV pump operations and including background information on medications and necessary volumetric calculations.

- An on-line assessment to allow students to test their knowledge and competency levels in a low stress environment.

- Provision of simple overall feedback as well as more detailed information over the course of the assessment to be able to be displayed. This should allow a thorough review of exactly where any mistakes are made during the assessment. These additional enhancements were assessed in the pilot study described in the following section.

\section{IV PUMP EMULATOR SYSTEM DESIGN}

The IVPE system comprises two distinct functional elements: A Programmable logic controller (PLC) to execute multiple parallel logic based functions; and a SCADA software system to deal with HMI tasks such as sense of visual and audio reality. The emulator system has been expanded from the original role of simply mimicking an IV infusion pump and is divided into three main sections:

- Learning Mode

- Resource Provision

- Assessment Mode

The Learning or Demonstration mode is achieved using so called step - transition logic within the programmable controller. Each logical condition must be met before transitioning to the next functional stage within the sequence. The logical sequences control individual outputs which when combined form the driving logic for the emulator. Logical sequences can be interrupted at any stage to allow the user the flexibility of referring back to support material.

Scaffolding resource material as shown in Fig. 2, is viewed and run within the SCADA software runtime environment and therefore a high level of interactivity between the programmable controller and SCADA system is required. The SCADA package chosen for this system allows easy integration with the programmable controller at a number of levels. This easily allows the integration of the sequencing and audio-visual aspects of the emulator system.

In assessment mode students are provided with a number of lifelike scenarios or case studies as in Fig. 3, whereby both simple pump operation and clinical reasoning skills are able to be assessed. The individual actions right down to button presses and values calculated can be extracted from the HMI system, logically evaluated against a performance rubric and a summary of results displayed at the end of each assessment session. Figure 4 shows a summary screen indicating specific errors so students can self assess their session and modify their approach accordingly.

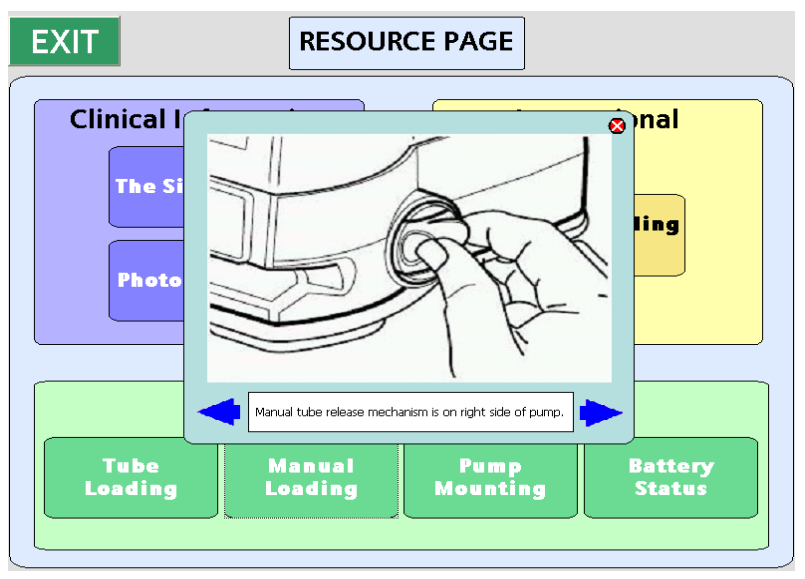

Figure 2. Additional scaffolding resource access .

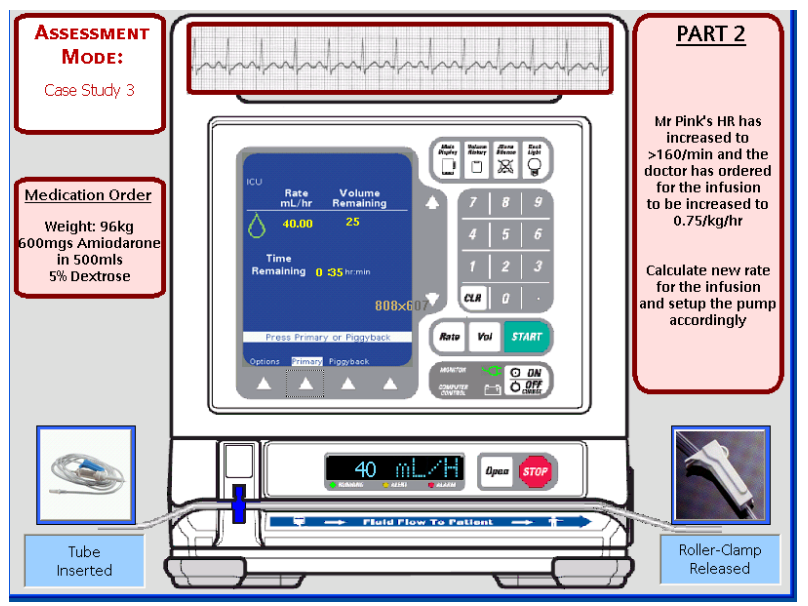

Figure 3. Typical Case study Screen

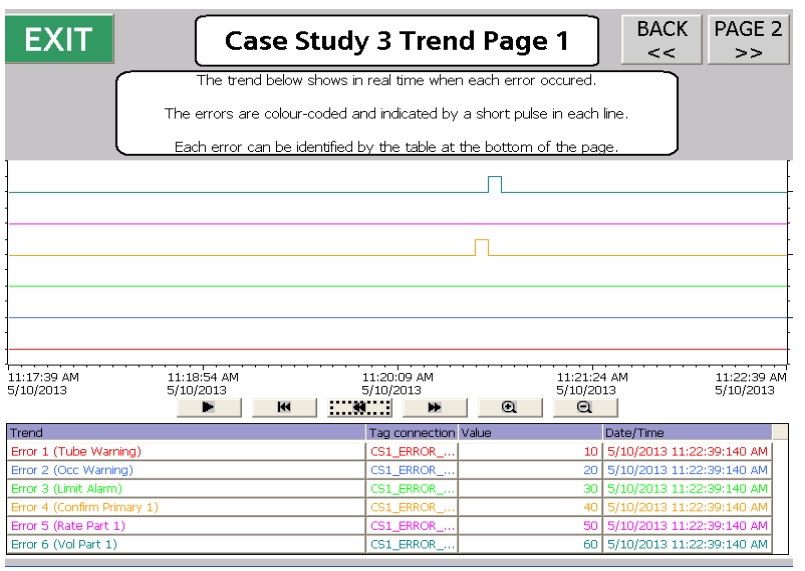

Figure 4. Case error evaluation chart

\section{A. Human Machine Interface Design Aspects}

In practice the main interactive element for the clinical nursing student is the IV infusion pump HMI. As medical instruments require effective use when the operator is using gloves the interface is not a touch-enabled device and is less intuitive than modern touch screen devices. In this case access to screen elements is via a membrane keypad with numeric entry keypad for entry of flow-rate and volumetric data. The SCADA system software [34] allows interaction via the same style keypad, buttons and lights as the real system. Where possible the exact functionality of the IV infusion pump is mimicked by the 
SCADA system, including aspects such as sequence timing, visual and audible cues and warnings. The only exception to this approach was with the overall infusion timeframe which may take hours in practice.

\section{B. Emulator Access}

Students at USQ are able to access the IVPE using the existing RAL system [19]. The system allows access to experiment bookings through the Learning Management System with which the students are already familiar. The students are also able to use the system in the traditional group situation on-campus during residential school.

\section{Methodology}

In the initial phase of the project student understanding was evaluated with 2 hour observations of classes. This included the way students and teachers use the IV pump emulator for learning and teaching with data collection via a student focus group, both done by external consultants. The focus group's aim was to gain insight into what students do to acquire the target skill and what their understanding of the skill is. The information gathered by the focus groups was used to enhance the features of the IVPE system and also contributed to the evaluation design for the second phase of the project.

\section{EVALUATION}

The IVPE was evaluated objectively to assess its effectiveness as part of the RAL based enhanced learning system during Semester 2, 2012. A group of 58 students undertaking the course, NUR2000 Medications in Nursing Practice, were recruited and allocated to one of three groups. Group 1 participants $(n=18)$ were from an online-only cohort of Bachelor of Nursing students. The remaining students were allocated randomly to an oncampus only group (Group $2 ; n=20$ ) or an on-campus + online group (Group 3; $n=20$ ).

Group 1 participants were involved in online-only training of the IVPE. Learning outcomes were assessed during a residential school on their performance using an actual IV pump, prior to any face to face instruction during class time. Group 2 participants were on-campus students who used the actual IV pump in laboratory classes and were not given access to the IVPE as an additional learning resource. Group 3 participants were also on-campus students who used the actual IV pump in class, but who were offered access to the IVPE as an additional learning resource. Group 2 and Group 3 participants were involved in training in class using the actual IV pump for a 2-hour period. Group 1 participants were encouraged to use the IVPE as often as possible, but for a period not exceeding two hours in total. The period of time for which each participant used the IVPE prior to attending residential school was recorded, with times ranging from 20 minutes to two hours.

Despite several attempts to engage and encourage Group 1 participants to use the IVPE, only six performed the initial login to register to use the RAL program and just four participants completed the training using the IVPE and were subsequently assessed using the actual pump during the residential school.

During the assessment, participants performed a series of activities individually, including turning the pump on and off, loading the IV tubing into the pump, and programming different rates and volumes of IV fluid from problem-based medication calculations. Sessions were timed and performance scores were allocated for each activity. The total number of points available was 130 . Ten participants from Group 2 and six participants from Group 3 completed all aspects of the training and subsequent assessment, leaving data for a total of 20 participants to go forward for analysis.

Given the small group sizes, probability analysis was augmented with calculation of effect sizes, using Cohen's $d$, to assess the meaningfulness of between-group differences. As a rule of thumb, a Cohen's $d$ value of 0.2 is considered small, 0.5 is considered moderate, and 0.8 is considered large.

Complete details of the results of the evaluation study are reported in [20]. The findings include:

- Group 3 (on-campus + online) practiced for longer ( $M=124$ mins) than the other two groups (Group 1: $M=65$ mins, $d=0.83$; Group 2: $M=44$ mins, $d=$ $1.21, p<.05)$.

- Group 1 (online only) took longer to complete the assessment $(M=837 \mathrm{secs})$ than the other two groups (Group 2: $M=722 \mathrm{secs}, d=1.41$; Group 3: $M=786$ secs, $d=0.47$ ).

- In terms of assessment, Group 3 scored, on average, more than 6 points higher $(M=119.0)$ than the other two groups (Group 1: $M=112.5, d=1.02$; Group 2: $M=112.8, d=0.80)$.

- For participants overall, a significant positive correlation was found between practice time and assessment scores $(r=.57, p=.009)$.

- No correlation was found between practice time and time to complete assessment $(r=.01, p=.98)$.

- A significant inverse correlation was found between time to complete assessment and assessment scores achieved $(r=-.49, p=.029)$.

These results suggest several things. Firstly, Group 3, who had access to both the IVPE and the actual pump, practiced for longer and performed better in their assessments than the other two groups. Indeed, Group 3 practiced on the IVPE $(M=89$ mins) for longer than Group 1 $(M=65$ mins, $d=0.20)$, who had access only to the IVPE. Secondly, lack of familiarity with the actual pump, as with Group 1, was associated with taking longer to complete the assessment. Thirdly, higher assessment scores were associated with (a) longer practice times, and (b) access to both the IVPE and the actual pump. Fourthly, the performance of Group 1 and Group 2 were essentially the same. Both of these groups had access to only one form of the IV pump (IVPE or actual pump). Overall, results showed that the IVPE was associated with similar outcomes to the traditional instruction in using the actual pump in a classroom setting, whereas those participants who were afforded access to the IVPE in addition to the actual IV pump in the classroom produced the best outcomes of the three groups. A follow-up evaluation using larger groups of undergraduate nursing students is scheduled for the latter half of 2013.

\section{FINDINGS}

Around the clock access, Australia wide means students can safely update or refresh their knowledge just prior to clinical placement in real-life situations. Access to the training system is on a one to one basis, where there is no 


\section{SPECIAL FOCUS PAPER \\ INTERACTIVE EMUlator SySTEM TO Aid CliniCAL PRACTICE OUTCOMES FOR NuRSES}

risk of a dominant group member 'taking over'. On the other hand should a student require additional information or a guided tour of the experiment this is now also possible.

In regards to the original research question of how well an emulated RAL IV pump can support skill acquisition these initial results suggest that there are no notable differences between the real practical learning activity and the emulator based learning activity. In fact they demonstrate that the best learning outcomes are achieved if both the online and face-to-face activities are done by students

\section{CONCLUSIONS}

This paper has described the second phase of a collaborative project between the Department of Nursing and Midwifery and the Faculty of Engineering to enhance RAL based Clinical Practice activities for nursing education. An enhanced prototype of a remotely accessible infusion pump emulator has been designed and implemented.

Initial feedback is very positive and shows strong justification for the use of an emulator to assist students in their clinical practice studies. The added enhancements to the original experimental design [3] have been received well by students. Of particular note is the summary feedback provided at the end of an assessment mode session as in Fig. 5. This appears to have increased student confidence which in turn has translated into improved clinical competency measures.

The IVPE evaluation has highlighted issues regarding ease of emulator access and booking system complexity. From an economic perspective the IVPE evaluation has shown significant cost savings are achievable for experiments of this nature. As an example the cost of expanding the emulator system to cater for 300 or more students is expected to be far less than the equivalent outlay for the equivalent physical experiment setup as used in the pilot project [3]. At the same time student feedback regarding the enhanced IVPE system useability has exceeded previous good results from [3]. It would appear that in the context of digitally controlled experimentation with fixed degrees of freedom, remote emulation combined with traditional physical labs appears to be an improvement on just being there. In addition, given these positive outcomes, further evaluation of clinical performance of using an IV pump at a patient's bedside in a simulated ward environment is recommended for future research.

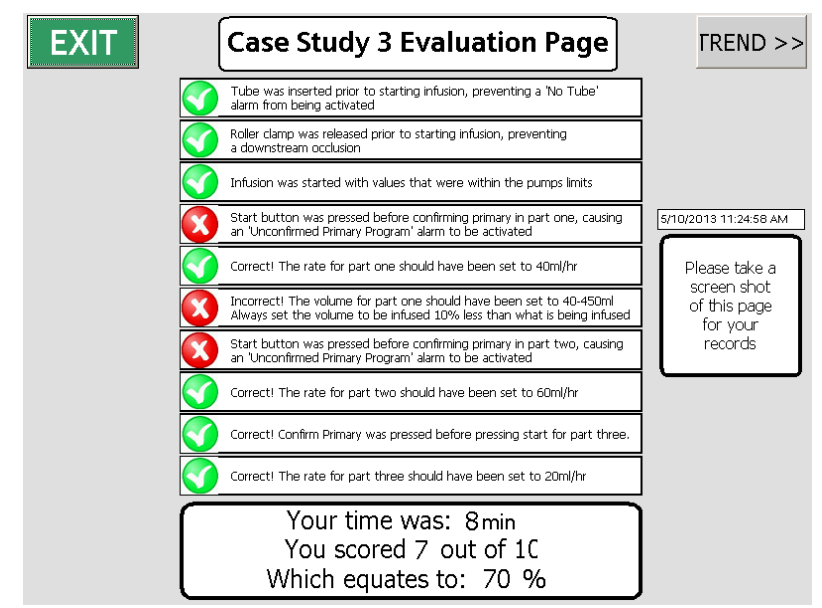

Figure 5. Summary Feedback in assessment mode

\section{ACKNOWLEDGEMENT}

The authors would like to thank Lesley Jolly and Hannah Jolly for their assistance with the evaluation and the Associate Deans (Learning and Teaching) at USQ for their support for this research project. This work was supported by a USQ Learning and Teaching Performance Fund Grant.

\section{REFERENCES}

[1] G. V. Kondraske, R. A. Volz, D. H. Johnson, D. Tesar, J. C. Trinkle, and C. R. Price, "Network-based infrastructure for distributed remote operations and robotics research," Robotics and Automation, IEEE Transactions on, vol. 9, pp. 702-704, 1993. http://dx.doi.org/10.1109/70.258062

[2] B. Aktan, C. A. Bohus, L. A. Crowl, and M. H. Shor, "Distance learning applied to control engineering laboratories," Education, IEEE Transactions on, vol. 39, pp. 320-326, 1996. http://dx.doi.org/10.1109/13.538754

[3] L. Bowtell, C. Moloney, A. A. Kist, V. Parker, A. Maxwell, and N. Reedy, "Enhancing Nursing Education with Remote Access Laboratories " International Journal of Online Engineering (iJOE), vol. 8, 2012.

[4] L. Bowtell, A. A. Kist, D. Osbourne, and V. Parker, "Improving Clinical Practice Outcomes for Nurses with an Interactive Emulator," presented at the IEEE EDUCON2013, Berlin, Germany, 2013.

[5] A. A. Kist, A. Maxwell, and P. Gibbings, "Expanding the Concept of Remote Access Laboratories " presented at the 119th ASEE Annual Conference and Exposition, San Antonio, Texas, 2012.

[6] R. T. White, "The link between the laboratory and learning," International Journal of Science Education, vol. 18, pp. 761 - 774, 1996. http://dx.doi.org/10.1080/0950069960180703

[7] M. Barak, "Instructional principles for fostering learning with ICT: teachers' perspectives as learners and instructors," Education and Information Technologies, vol. 11, pp. 121-135, 2006. http://dx.doi.org/10.1007/s11134-006-7362-9

[8] L. A. M. P. Slangen and P. B. Sloep, "Mind tools contributing to an ICT-rich learning environment for technology education in primary schools," International Journal of Continuing Engineering Education and Life Long Learning, vol. 15, pp. 225239, 2005. http://dx.doi.org/10.1504/IJCEELL.2005.007712

[9] D. H. Jonassen, Computers as mindtools for schools: engaging critical thinking: Merrill, 2000.

[10] D. Jonassen, C. Carr, and H.-P. Yueh, "Computers as mindtools for engaging learners in critical thinking," TechTrends, vol. 43, pp. 24-32, 1998. http://dx.doi.org/10.1007/BF02818172

[11] T. Levett-Jones, et al, "'The 'five rights' of clinical reasoning: An educational model to enhance nursing students' ability to identify and manage clinically 'at risk' patients'," Nurse Education Today, vol. 30, pp. 515-520, 2010. http://dx.doi.org/10.1016/j.nedt.2009. 10.020

[12] N. Harder, "Use of Simulation in Teaching and Learning in Health Sciences: A Systematic Review," Journal of Nursing Education, vol. 49, pp. 23-28, 2010. http://dx.doi.org/10.3928/0148483420090828-08

[13] T. Pike and V. O'Donell, "The impact of clinical simulation on learner self-efficacy in pre-registration nursing education," Nurse Education Today, 2010. http://dx.doi.org/10.1016/j.nedt.2009. 09.013

[14] P. Messmer, "Enhancing Nurse-Physician Collaboration Using Pediatric Simulation," Journal of Continuing Education in Nursing, vol. 39, 2008.

[15] Rauen, "Simulation as a Teaching Strategy for Nursing Education and Orientation in Cardiac Surgery " Critical Care Nurse, vol. 24, pp. 46-51, 2004.

[16] E. Bandman, Bandman, B, "Critical Thinking in Nursing," 1995.

[17] D. Sampsel, G. Bharwani, D. Mehling, and S. Smith, "Robots as Faculty: Student and Faculty Perceptions," Clinical Simulation in Nursing, vol. In Press, Corrected Proof, 2010.

[18] Medical_News_Today, "Robot Joins Nursing Institute Of West Central Ohio To Address Faculty Shortage," 2007. 
[19] A. A. Kist and P. Gibbings, "Inception and management of remote access laboratory projects," presented at the AaeE 2010: 21st Annual Conference of the Australasian Association for Engineering Education, Sydney, Australia, 2010.

[20] V. R. Parker, P. C. Terry, C. Moloney, and L. Bowtell, "Evaluation of an online emulated intravenous pump for nursing students," Journal of Clinical Nursing [under review], 2013.

\section{AUTHORS}

Les Bowtell, Alexander A. Kist, and Daniel Osbourne are with the Faculty of Engineering and Surveying, University of Southern Queensland, Toowoomba, Australia.

Victoria R. Parker is with the Faculty of Science, University of Southern Queensland, Toowoomba, Australia.

This article is an extended and modified version of a paper presented at the EDUCON2013 conference held at Technische Universität Berlin, Berlin, Germany from March 13-15, 2013. Received 15 May 2013. Published as resubmitted by the authors 27 May 2013. 